\title{
Prenatal management of fetal supravevtricular tachycardia
}

\author{
Rasanjana DPL, Dias T
}

\begin{abstract}
Introduction: Fetal cardiac arrhythmias are discovered in about $1 \%$ of fetuses and most of them are supraventricular tachycardia (SVT). Persistent tachycardia with a fetal heart rate (FHR) more than 220 bpm could lead into congestive cardiac failure and can manifests as non-immune fetal hydrops. With the rapid advancement in fetal echocardiographic skills, such arrhythmias can be diagnosed accurately during prenatal life by using M-mode echocardiogram and Doppler.
\end{abstract}

Case 1: A 28 year old primigravida was referred from Jaffna due to fetal tachycardia and ascites detected at routine scan at 27 weeks. Detailed anomaly scan including cardiac evaluation was performed after admission to our unit and SVT with a heart rate of $278 \mathrm{bpm}$ with hydrops fetalis found with no other gross anomalies. Mother was started on digoxin $0.25 \mathrm{mg}$ twice a day and changed in to sotalol $80 \mathrm{mgtwice}$ a day after 48 hours. Sotalol omitted and flicanide $100 \mathrm{mg}$ twice a day started after 4days. Because of persistently high

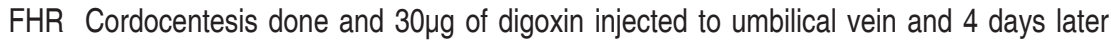
amioderone $5 \mathrm{mg}$ injected. Subsequently FHR decreased to $137 \mathrm{bpm}$.

Case 2: A 38 year old, mother of one was transferred from Badulla due to fetal tachycardia at 36 weeks. Scan at our unit revealed Ebstein anomaly of fetus lead to re-entral tachycardia. Atrial rate was $338 \mathrm{bpm}$ and ventricular rate was $168 \mathrm{bpm}$. Baby delivered electively and admitted to SCBU.

Discussion: Fetal SVTs may be associated with adverse perinatal outcome. Development of fetal hydrops is a poor prognostic factor and placental oedema reduces transfer of drugs to fetus. Early identification and intervention (before development of hydrops) will lead to better perinatal outcome.

\section{INTRODUCTION}

Fetal cardiac arrhythmias are discovered in about $1 \%$ of fetuses and most of them are supraventricular tachycardia (SVT). ${ }^{1}$ Sustained SVT with a fetal heart rate (FHR) more than $220 \mathrm{bpm}$ may lead to congestive cardiac failure and can manifests as non-immune fetal hydrops. ${ }^{2}$ With the rapid advancement in fetal echocardiographic equipments and techniques, such arrhythmias can be

Professorial Obstetrics and Gynaecology Unit, Faculty of Medicine, University of Kelaniya, Sri Lanka

Correspondence: Dr.Tiran Dias

E-mail: thiran_dias@yahoo.com

Competing interests: None diagnosed accurately during prenatal life. In utero management of fetal SVT with fetal hydropsis challenging because transplacental drug transfer decreases as a result of placental oedema. ${ }^{3}$ There is no clear consensus regarding the best drug regimen for fetal SVT. Digoxin is considered as the first line drug in the treatment and maternally administered digoxin is usually successful in controlling tachycardia in non hydrophic fetus. Sotalol and flicanide are used as second line agents. Cordocentesis enables direct access to the fetal circulation and may allow a quicker response to therapy. Here we report a case of fetal SVT with hydrops detected at 29 weeks and managed with combination of oral and inrta umbilical drugs and a case with reentrant tachycardia due to Ebstien anomaly.

\section{CASE 01}

A 28 year old primigravida at 29 weeks was referred to our unit from Manipai, Jaffna due to fetal tachycardia and ascites detected at routine scan. In her antenatal history, booking visit is at 7 weeks of gestation and booking investigations were normal. No maternal or family history of cardiac diseases and no history of fever, intake of drugs or thyrotoxicosis.

First ultrasound scan (USS) done at 13 weeks (dating scan) and not mentioned about fetal anomalies. After admission to our unit detailed anomaly scan performed including cardiac evaluation and it was found to be within normal limits. The four chamber view of the heart was normal. Most important finding was SVT with a heart rate of $278 \mathrm{bpm}$ with ascites.

Medical cardio version of the fetus was decided and mother was started with oral digoxin $0.25 \mathrm{mg}$ twice a day and continued for 48 hours. Matermal heart rate and serum electrolytes were monitored. After 48 hours fetal heat rate (FHR) was remained at $230 \mathrm{bpm}$. An opinion from a consultant cardiac electro-physiology was sought. Digoxin was omitted and oral Sotalol $80 \mathrm{mg}$ twice a day was started. FHR has reduced to $130 \mathrm{bpm}$ initially but started to rise by fourth day of therapy and FHR raised up to $262 \mathrm{bpm}$. Later it was decided to omit Sotalol and commence Flicanide 100mg twice a day. Despite of maximum therapeutic dose of Flicanide FHR did not reduce and fetal hydrops was progressively worsen. Consent has been obtained from the mother for cordocentesis and intra-umbilival vein Digoxin $30 \mu \mathrm{g}$ was administered. Subsequently FHR decreased to $137 \mathrm{bpm}$ and maternal oral 0.25 of digoxin twice a day continued. After three days FHR raised up to $268 \mathrm{bpm}$. Subsequently, intra-umbilical vein Amioderone 5mg 
was injected and oral Amioderone $200 \mathrm{mg}$ twice a day was added. Two days later mother complained of reduced fetal movements and USS reveled death in utero of fetus.

\section{CASE 02}

A 38 year old mother of one was referred to our unit from General Hospital Badulla due to fetal tachycardia at 36 weeks. She booked at 8 weeks of gestation and booking investigations were normal. She attended for first ultrasound at 16 weeks. Antenatal history was uneventful till 36 weeks and found abnormal fetal heart rate pattern and referred to our unit. Detailed anomaly scan performed including cardiac evaluation and it revealed Ebstein anomaly of fetus lead to re-entral tachycardia. Atrial rate was $338 \mathrm{bpm}$ and ventricular rate was $168 \mathrm{bpm}$. Since fetus is at reasonable maturity, immediate delivery was decided.

Baby girl was delivered following a caesarean section and admitted to SCBU. Cardiac evaluation done with ECG and 2D echocardiogram and it confirmed the prenatal diagnosis of Ebstein anomaly.

\section{DISCUSSION}

Fetal SVT is the most commonly encountered fetal cardiac arrhythmia in pregnancy that may be associated with adverse perinatal out come. ${ }^{4}$ Maternal factors such as excessive consumption of caffeine, smoking, illicit drugs and fetal anomalies such as fetal cardiac malformations, extra cardiac malformations like diaphragmatic hernia may contribute to development of SVT. ${ }^{5}$ The predominant mechanism of developing SVT in fetus is believed to be due to atrio-ventricular reentrant tachycardia. ${ }^{6,7}$ With advances in ultrasound technology it is now possible to diagnose accurately the type of arrhythmia using M-mode echocadiogram to compare the relationship between atrial and ventricular contractions. ${ }^{7}$

SVT complicated with fetal hydrops could be managed either by in utero therapy if the fetus is too premature or by immediate delivery if the pregnancy is of reasonable maturity in particular if beyond 34 weeks. In our two cases, one managed in utero and other one delivered immediately. Digoxin remains the drug of first choice and it terminates circular movement within re-entrant circuits by prolonging the refractory phase. ${ }^{4}$ Sotalol produce an increase in the duration of the action potential and lengthen the effective and absolute refractory period. ${ }^{4}$ Flicanide depresses conduction throughout the myocardium and prolongs refractory period. ${ }^{4}$

Development of fetal hydrops is a poor prognostic factor. Placental oedema reduces transfer of drugs to fetus. There are several methods to bypass placenta and administer drugs to fetus such as intra-umbilical, intra-amniotic, intra-peritoneal, intramuscular and intra-cardiac. Most of these invasive measures need expertise and carry significant risk to the fetus.

\section{REFERENCES}

1. Parilla B, Strasburger J,Fetal arrhythmias, Global library of women's medicine.2008

2. Strasburger JF, Cheulkar B, Wichman HJ. Perinatal arrhythmias: Diagnosis and management. Clinics in Perinatology 2007; 34:627-52.

3. Perles Z, Gavri S, Rein AJTT. Tachyarrhythmias in the fetus: State of the art diagnosis and treatment. Progress in Pediatric Cardiology 2006: 22; 95-107.

4. Rana YS, Sodhi B, Kochar SPS, Arora $D$, Successful digoxin therapy for fetal supraventricular tachycardia, South Asian journal of obstetrics and gynaecology. January-April 2009; 1(1): 44-46

5. Tulzer $G$, Khowsathit $P$, Gudmundsson $S$, et al: Diastolic function of the fetal heart during second and third trimester: A prospective longitudinal Dopplerechocardiographic study. European journal of pediatrics. 1994; 151-153

6. Bergman $G$, Jacobsson $L A$, Wahren-Herlenius $M$ et al: Doppler echocardiographic and electrocardiographic atrioventricular timeintervals in newborn infants: evaluation of techniques for surveillance offetuses at risk for congenital heart block. Ultrasound in obstetrics and gynaecology. 2006; 28(1): 57-62

7. Yam MC, Leuna JY, Suna RYT, A fetus with hydropic changes secondary to fetal SVT, Hongkong journal of pediatrics (new series)2003;8:130-132 